ELECTRONIC RESEARCH ANNOUNCEMENTS OF THE AMERICAN MATHEMATICAL SOCIETY

Volume 10, Pages 29-38 (April 6, 2004)

S $1079-6762(04) 00127-1$

\title{
HOMEOMORPHISM GROUPS OF MANIFOLDS AND ERDŐS SPACE
}

\author{
JAN J. DIJKSTRA AND JAN VAN MILL
}

(Communicated by Krystyna Kuperberg)

\begin{abstract}
Let $M$ be either a topological manifold, a Hilbert cube manifold, or a Menger manifold and let $D$ be an arbitrary countable dense subset of $M$. Consider the topological group $\mathcal{H}(M, D)$ which consists of all autohomeomorphisms of $M$ that map $D$ onto itself equipped with the compact-open topology. We present a complete solution to the topological classification problem for $\mathcal{H}(M, D)$ as follows. If $M$ is a one-dimensional topological manifold, then $\mathcal{H}(M, D)$ is homeomorphic to $\mathbb{Q}^{\infty}$, the countable power of the space of rational numbers. In all other cases we found that $\mathcal{H}(M, D)$ is homeomorphic to the famed Erdős space $\mathfrak{E}$, which consists of the vectors in Hilbert space $\ell^{2}$ with rational coordinates. We obtain the second result by developing topological characterizations of Erdős space.
\end{abstract}

\section{INTRODUCTION}

All spaces under discussion are separable and metrizable. If $X$ is compact, then the standard topology on the group of homeomorphisms $\mathcal{H}(X)$ of $X$ is the so-called compact-open topology (which coincides with the topology of uniform convergence). This topology makes $\mathcal{H}(X)$ a Polish topological group. For locally compact spaces, the compact-open topology is Polish but not necessarily a group topology. We therefore think of $X$ as a subspace of its Alexandroff one-point compactification $\alpha X=X \cup\{\infty\}$, and we topologize $\mathcal{H}(X)$ by identifying it with the closed subgroup $\{h \in \mathcal{H}(\alpha X): h(\infty)=\infty\}$ of $\mathcal{H}(\alpha X)$. If every point in $X$ has a neighbourhood that is a continuum, then the just described topology on $\mathcal{H}(X)$ coincides with the compact-open topology; see Dijkstra [11] and Arens [3]. If $A$ is a subset of a space $X$, then $\mathcal{H}(X, A)$ stands for the subgroup $\{h \in \mathcal{H}(X): h(A)=A\}$ of $\mathcal{H}(X)$.

Brouwer [7] showed that $\mathbb{R}$ is countable dense homogeneous, that is, for all countable dense subsets $A$ and $B$ of $\mathbb{R}$ there is an $h \in \mathcal{H}(\mathbb{R})$ with $h(A)=B$. It is not difficult to prove that every $\mathbb{R}^{n}$ has this property. In view of Brouwer's result it is a natural idea to investigate the group $\mathcal{H}\left(\mathbb{R}^{n}, \mathbb{Q}^{n}\right)$. It was shown in Dijkstra and van Mill 12 that the group $\mathcal{H}(\mathbb{R}, \mathbb{Q})$ is homeomorphic to the zero-dimensional space $\mathbb{Q}^{\infty}$, the countable infinite product of copies of the rational numbers $\mathbb{Q}$. In contrast, we showed in [12] (see also [10]) that $\mathcal{H}\left(\mathbb{R}^{n}, \mathbb{Q}^{n}\right)$ for $n \geq 2$ contains a closed copy of the famed Erdős space $\mathfrak{E}$ which is known to be one-dimensional; see 17. This result led us to consider the question whether $\mathcal{H}\left(\mathbb{R}^{n}, \mathbb{Q}^{n}\right)$ (for $n \geq 2$ ) is in fact homeomorphic to Erdős space. We announce here that it is. We prove that

Received by the editors September 30, 2003.

2000 Mathematics Subject Classification. Primary 57S05. 
if $D$ is a countable dense subset of a locally compact space $X$, then $\mathcal{H}(X, D)$ is an Erdős space factor, which means that $\mathcal{H}(X, D) \times \mathfrak{E}$ is homeomorphic to $\mathfrak{E}$. Under rather mild extra conditions, the group $\mathcal{H}(X, D)$ is found to be homeomorphic to Erdős space. This is the case if $X$ contains a nonempty open subset homeomorphic to $\mathbb{R}^{n}$ for $n \geq 2$, an open subset of the Hilbert cube $Q$, or an open subset of some universal Menger continuum. As an application it follows that if $M$ is an at least 2-dimensional manifold (with or without boundary) and $D$ is a countable dense subset of $M$, then $\mathcal{H}(M, D)$ is homeomorphic to Erdős space.

Homeomorphism groups of manifolds are very well studied. Let $\mathbb{I}$ denote the interval $[0,1]$ and let $\mathcal{H}_{\partial}\left(\mathbb{I}^{n}\right)$ stand for the subgroup of $\mathcal{H}\left(\mathbb{I}^{n}\right)$ consisting of homeomorphisms that fix the boundary of the $n$-cube $\mathbb{I}^{n}$. Anderson [2] proved that $\mathcal{H}_{\partial}(\mathbb{I})$ is homeomorphic to the separable Hilbert space $\ell^{2}$ (see [4, Proposition VI.8.1] or 20]). It was shown by Luke and Mason [23] that $\mathcal{H}_{\partial}\left(\mathbb{I}^{2}\right)$ is an absolute retract, which implies that $\mathcal{H}_{\partial}\left(\mathbb{I}^{2}\right) \approx \ell^{2}$ (apply for instance Dobrowolski and Toruńczyk [15]). For $n \geq 3$ it is open whether $\mathcal{H}_{\partial}\left(\mathbb{I}^{n}\right)$ is an absolute retract. This is one of the most interesting open problems in infinite-dimensional topology. For the Hilbert cube $Q$, that is, for $n=\infty$, the analogous problem was solved by Ferry [18] and Toruńczyk [28]. They proved that $\mathcal{H}(Q)$ is homeomorphic to $\ell^{2}$ (observe that $Q$ has no boundary). For $3 \leq n<\infty$ it is unknown what the topological classification of $\mathcal{H}_{\partial}\left(\mathbb{I}^{n}\right)$ or $\mathcal{H}\left(\mathbb{I}^{n}\right)$ is. By our results, the subgroups $\mathcal{H}_{\partial}\left(\mathbb{I}^{n},(\mathbb{Q} \cap \mathbb{I})^{n}\right)$ and $\mathcal{H}\left(\mathbb{I}^{n},(\mathbb{Q} \cap \mathbb{I})^{n}\right)$ are known; they are homeomorphic to Erdős space.

Recall that the Erdös space $\mathfrak{E}$ is the 'rational Hilbert space', that is the set of vectors in $\ell^{2}$ the coordinates of which are all rational. This space was introduced by Hurewicz who asked to compute its dimension. Erdős [17] proved that $\mathfrak{E}$ is onedimensional by establishing that every nonempty clopen subset of $\mathfrak{E}$ is unbounded. This result, in combination with the obvious fact that $\mathfrak{E}$ is homeomorphic to $\mathfrak{E} \times \mathfrak{E}$, lends the space its importance in dimension theory. Complete Erdoss space $\mathfrak{E}_{\mathrm{c}}$ is the 'irrational Hilbert space', that is, the set of vectors in $\ell^{2}$ all coordinates of which are irrational. In contrast to $\mathfrak{E}$, the complete Erdős space is topologically complete, being a $G_{\delta}$-subset of $\ell^{2}$. The space $\mathfrak{E}_{\mathrm{c}}$ surfaced in topological dynamics as the 'endpoint' set of several interesting objects. See Kawamura, Oversteegen, and Tymchatyn [19] for more information.

In order to prove our results we first present several increasingly powerful topological characterizations of Erdős space. What sets Erdős space apart from familiar spaces is that in addition to the one-dimensional topology that it inherits from $\ell^{2}$, an important role is played by the zero-dimensional topology that $\mathfrak{E}$ inherits from the product space $\mathbb{Q}^{\infty}$. This bitopological aspect prompted us to define and develop several new concepts in topology that link the two topologies in the characterization theorems. We demonstrate the power of our characterizations by deriving from them the above results with relative ease. Along the way, we get several other interesting results. For example, Erdős space is homeomorphic to its countable infinite power. Here we have a striking contrast with $\mathfrak{E}_{\mathrm{c}}$, which is not homeomorphic to $\mathfrak{E}_{\mathrm{c}}^{\infty}$, as was proved by Dijkstra, van Mill, and Steprāns [14]. In addition, Erdős space is homeomorphic to $\mathfrak{E}_{\mathrm{c}} \times \mathbb{Q}^{\infty}$, and every nonempty open subset of $\mathfrak{E}$ is homeomorphic to $\mathfrak{E}$.

We conclude with the observation that Erdös space started its career as a curious example in dimension theory. It turns out however that it is a fundamental object that surfaces in many places. In addition, it allows for a useful and easily applied 
topological characterization just as several other fundamental objects in topology: the Cantor set (Brouwer [6]), the Hilbert cube (Toruńczyk [29]), Hilbert space (Toruńczyk [30]), the universal Menger continua (Bestvina [5]), and the Nöbeling spaces (Ageev [1]).

Detailed proofs supporting the theorems we announce here will appear in [12] for $\S 2,[13$ ] for $\S \S 3-5$, and [10] for $\S 5$.

\section{The ZERO-DIMENSIONAL CASE}

In this section we consider $\mathcal{H}(M, D)$, where $M$ is a one-dimensional topological manifold. We recall the following characterization of the space $\mathbb{Q}^{\infty}$, which follows from a theorem of Steel [27]; see also van Engelen [16, Theorem A.2.5].

Theorem 2.1. A space $X$ is homeomorphic to $\mathbb{Q}^{\infty}$ if and only if $X$ is a zerodimensional, first category $F_{\sigma \delta}$-space with the property that no nonempty clopen subset is a $G_{\delta \sigma}$-space.

In particular, we have:

Corollary 2.2. If $X$ is a homogeneous, zero-dimensional, first category $F_{\sigma \delta}$-space that contains a closed copy of $\mathbb{Q}^{\infty}$, then $X$ is homeomorphic to $\mathbb{Q}^{\infty}$.

The following theorem is proved by constructing a closed imbedding of $\mathbb{Q}^{\infty}$ in $\mathcal{H}(\mathbb{R}, \mathbb{Q})$.

Theorem 2.3. $\mathcal{H}(\mathbb{R}, \mathbb{Q})$ is homeomorphic to $\mathbb{Q}^{\infty}$.

Corollary 2.4. Let $D$ be a countable dense subset of a locally compact space $X$. If $X$ contains an open set that is homeomorphic to $\mathbb{R}$, then $\mathcal{H}(X, D)$ is homeomorphic to $\mathbb{Q}^{\infty}$ if and only if $\mathcal{H}(X, D)$ is zero-dimensional.

We also showed that if $D$ is a countable dense subset of a Cantor set $C$, then $\mathcal{H}(C, D) \approx \mathbb{Q}^{\infty}$.

\section{Almost Zero-dimensional spaces}

Let $p \in(0, \infty)$ and consider the (quasi-)Banach space $\ell^{p}$. This space consists of all sequences $z=\left(z_{0}, z_{1}, z_{2}, \ldots\right) \in \mathbb{R}^{\infty}$ such that $\sum_{i=0}^{\infty}\left|z_{i}\right|^{p}<\infty$. The topology on $\ell^{p}$ is generated by the norm $\|z\|=\left(\sum_{i=0}^{\infty}\left|z_{i}\right|^{p}\right)^{1 / p}$. It is well known that the norm topology on $\ell^{p}$ is generated by the product topology (that is inherited from $\mathbb{R}^{\infty}$ ) together with the sets $\left\{z \in \ell^{2}:\|z\|<t\right\}$ for $t>0$. We extend the $p$-norm over $\mathbb{R}^{\infty}$ by putting $\|z\|=\infty$ when $z \in \mathbb{R}^{\infty} \backslash \ell^{p}$. Note also that the norm as a function from $\mathbb{R}^{\infty}$ to $[0, \infty]$ is not continuous because the norm topology is much stronger than the product topology, but that this function is lower semi-continuous (LSC). We define the Erdös space by

$$
\mathfrak{E}=\left\{z \in \ell^{2}: z_{i} \in \mathbb{Q} \text { for each } i\right\} .
$$

Let $\mathcal{T}$ stand for the zero-dimensional topology that $\mathfrak{E}$ inherits from $\mathbb{Q}^{\infty}$. Observe that $\mathcal{T}$ is weaker than the norm topology, and hence that $\mathfrak{E}$ is totally disconnected. We have by the remark above that the graph of the norm function, when seen as a function from $(\mathfrak{E}, \mathcal{T})$ to $\mathbb{R}^{+}=[0, \infty)$, is homeomorphic to $\mathfrak{E}$. So, informally, we can think of $\mathfrak{E}$ as a 'zero-dimensional space with some LSC function declared continuous'. We find it convenient to work with USC rather than LSC functions, and we therefore define $\eta: \mathbb{Q}^{\infty} \rightarrow \mathbb{R}^{+}$by $\eta(z)=1 /(1+\|z\|)$, where $1 / \infty=0$. 
There is an interesting connection between the two topologies on $\mathfrak{E}$ that we would like to draw attention to. Because the norm is LSC on $\mathbb{R}^{\infty}$, every closed $\varepsilon$-ball in $\mathfrak{E}$ is also closed in the zero-dimensional space $\mathbb{Q}^{\infty}$. Thus we have that every point in $\mathfrak{E}$ has arbitrarily small neighbourhoods which are intersections of clopen sets.

A subset $A$ of a space $X$ is called a $C$-set in $X$ if $A$ can be written as an intersection of clopen subsets of $X$. A space is called almost zero-dimensional if every point of the space has a neighbourhood basis consisting of C-sets of the space. This concept is due to Oversteegen and Tymchatyn [24. The definition we use here is different from the original one but its equivalence is established in Dijkstra, van Mill, and Steprāns [14]. Note that almost zero-dimensionality is hereditary. It is proved in 24] that every almost zero-dimensional space is at most one-dimensional; see also Levin and Pol 22 .

Thus $\mathfrak{E}$ is almost zero-dimensional. In fact, it is a universal object for the class of almost zero-dimensional spaces (in contrast, the class of totally disconnected spaces has no universal element; see Pol [25]):

Theorem 3.1. The following statements about a space $X$ are equivalent:

(1) $X$ is almost zero-dimensional,

(2) $X$ is homeomorphic to the graph of some USC or LSC function with a domain of dimension at most zero,

(3) $X$ is imbeddable in complete Erdös space $\mathfrak{E}_{\mathrm{c}}$, and

(4) $X$ is imbeddable in Erdös space $\mathfrak{E}$.

This theorem can be extracted from results in the papers 24] and 19]. Particularly important is the characterization theorem in 24 that states that a space is almost zero-dimensional if and only if it is homeomorphic to the set of endpoints of some $\mathbb{R}$-tree. As a corollary to Theorem 3.1 we have that the nonempty C-sets in an almost zero-dimensional space are precisely the retracts of the space.

If $Z$ is a set that contains $X$, then we say that a (separable metric) topology $\mathcal{T}$ on $Z$ witnesses the almost zero-dimensionality of $X$ if $\operatorname{dim}(Z, \mathcal{T}) \leq 0, O \cap X$ is open in $X$ for each $O \in \mathcal{T}$, and every point of $X$ has a neighbourhood basis in $X$ consisting of sets that are closed in $(Z, \mathcal{T})$. We will also say that the space $(Z, \mathcal{T})$ is a witness to the almost zero-dimensionality of $X$. The archetype is $\mathbb{Q}^{\infty}$ as a witness to the almost zero-dimensionality of Erdős space.

\section{Characterizing Erdős space topology}

Let $\varphi, \psi: X \rightarrow \mathbb{R}^{+}$be such that $\psi(x) \leq \varphi(x)$ for all $x \in X$. We define

$$
G_{\psi}^{\varphi}=\{(x, \varphi(x)): x \in X \text { and } \varphi(x)>\psi(x)\}
$$

and

$$
L_{\psi}^{\varphi}=\{(x, t): x \in X \text { and } \psi(x) \leq t \leq \varphi(x)\},
$$

both equipped with the topology inherited from $X \times \mathbb{R}^{+}$. Observe that $G_{0}^{\eta}$ is homeomorphic to $\mathfrak{E}$.

We say that $\varphi$ is a Lelek function with bias $\psi$ if $X$ is zero-dimensional, $\varphi$ and $\psi$ are USC, $X^{\prime}=\{x \in X: \psi(x)<\varphi(x)\}$ is dense in $X$, and $G_{\psi}^{\varphi}$ is dense in $L_{\psi \mid X^{\prime}}^{\varphi \mid X^{\prime}}$ If $\varphi$ is a Lelek function with bias 0 , then $\varphi$ is simply called a Lelek function. This terminology finds its origin in the following fact. If $\varphi$ is a Lelek function with compact domain $C$, then we obtain a Lelek fan [21] by identifying the base $C \times\{0\}$ in $L_{0}^{\varphi}$ to a point. Observe that $\eta$ is a Lelek function. 
If $A$ is a nonempty set, then $A^{<\omega}$ denotes the set of all finite strings of elements of $A$, including the null string $\lambda$. Let $A^{\omega}$ denote the set of all infinite strings of elements of $A$. If $s \in A^{<\omega}$ and $\sigma \in A^{<\omega} \cup A^{\omega}$, then we put $s \prec \sigma$ if $s$ is an initial substring of $\sigma$. If $\sigma \in A^{<\omega} \cup A^{\omega}$ and $k \in \omega$, then $\sigma\left\lceil k \in A^{<\omega}\right.$ is the string of length $k$ with $\sigma\left\lceil k \prec \sigma\right.$. A tree $T$ over a set $A$ is a subset of $A^{<\omega}$ that is closed under initial segments, that is, if $s \in T$ and $t \prec s$, then $t \in T$. An infinite branch of $T$ is an element $\sigma$ of $A^{\omega}$ such that $\sigma\lceil k \in T$ for every $k \in \omega$. The body of $T$, written as $[T]$, is the set of all infinite branches of $T$. If $s \in T$, then $\operatorname{succ}(s)$ denotes the set of immediate successors of $s$ in $T$.

Sierpiński [26] has shown that $X$ is an (absolute) $F_{\sigma \delta}$-space if and only if there exists a nonempty tree $T$ over a countable set and closed subsets $X_{s}$ of $X$ for each $s \in T$ such that:

i. $X_{\lambda}=X$ and $X_{s}=\bigcup\left\{X_{t}: t \in \operatorname{succ}(s)\right\}$ for all $s \in T$ and

ii. if $\sigma \in[T]$, then the sequence $X_{\sigma\lceil 0}, X_{\sigma\lceil 1}, \ldots$ converges to a point $x_{\sigma} \in X$.

Let us call such a system $\left(X_{s}\right)_{s \in T}$ a Sierpinski stratification of $X$. Van Engelen [16. Theorem A.1.6] has shown that a zero-dimensional space $X$ is homeomorphic to $\mathbb{Q}^{\infty}$ if there exists a Sierpiński stratification $\left(X_{s}\right)_{s \in T}$ of $X$ such that $X_{t}$ is nowhere dense in $X_{s}$ whenever $t \in \operatorname{succ}(s)$. Our characterizations of $\mathfrak{E}$ were inspired by these results.

Definition 4.1. SL is the class of all bounded USC functions $\varphi: X \rightarrow \mathbb{R}^{+}$such that $X$ is a zero-dimensional space for which there exists a Sierpiński stratification $\left(X_{s}\right)_{s \in T}$ with the following properties:

(a) if $s \in T$ and $t \in \operatorname{succ}(s)$, then $G_{0}^{\varphi \uparrow X_{t}}$ is nowhere dense in $G_{0}^{\varphi \uparrow X_{s}}$ and

(b) if $s \in T$, then $\varphi\left\lceil X_{s}\right.$ is a Lelek function.

If we define $T=\mathbb{Q}^{<\omega}$ and $X_{q_{1} \ldots q_{k}}=\left\{q_{1}\right\} \times \cdots \times\left\{q_{k}\right\} \times \mathbb{Q} \times \mathbb{Q} \times \cdots$, then it is a straightforward exercise to show that $\eta: \mathbb{Q}^{\infty} \rightarrow \mathbb{R}^{+}$is an element of SL. We call a pair $(h, \beta)$ a homeomorphism from a function $\varphi: X \rightarrow \mathbb{R}^{+}$to a function $\psi: Y \rightarrow \mathbb{R}^{+}$if $h: X \rightarrow Y$ is a homeomorphism and $\beta: X \rightarrow(0, \infty)$ is a continuous map with $\psi \circ h=\beta \cdot \varphi$.

Theorem 4.2. Any two elements of SL are homeomorphic and hence a space $E$ is homeomorphic to $\mathfrak{E}$ if and only if $E \approx G_{0}^{\varphi}$ for some $\varphi \in \mathrm{SL}$.

We sketch the method by which we proved this theorem. Let $\varphi: X \rightarrow \mathbb{R}^{+}$and $\psi: Y \rightarrow \mathbb{R}^{+}$be elements of $\mathrm{SL}$ and let $\left(X_{t}\right)_{t \in T}$ and $\left(Y_{s}\right)_{s \in S}$ be the associated Sierpiński stratifications. We construct a boolean algebra of clopen subsets of $X$ such that the associated Stone space $C$ is a compactification of $X$ that admits an extension $\tilde{\varphi}: C \rightarrow \mathbb{R}^{+}$of $\varphi$ with the property that $\tilde{\varphi} \mid \bar{X}_{t}$ is a Lelek function for each $t \in T$. The function $\psi$ is similarly extended to $\tilde{\psi}: D \rightarrow \mathbb{R}^{+}$. Van Engelen's proof [16. pp. 115-120] of the characterization of $\mathbb{Q}^{\infty}$ in terms of Sierpiński stratifications shows that there exists a homeomorphism $h: C \rightarrow D$ with $h(X)=Y$. This homeomorphism in general will not correspond to a homeomorphism between the functions $\tilde{\varphi}$ and $\tilde{\psi}$. In order to get a continuous $\beta: C \rightarrow(0, \infty)$ such that $\tilde{\psi} \circ h=\beta \cdot \tilde{\varphi}$ and $h(X)=Y$ we need to add an additional ingredient to van Engelen's construction as follows. The uniqueness of the Lelek fan as proved by Bula and Oversteegen [8] and Charatonik [9] allows us to develop an 'unknotting' theory for Lelek functions which does the trick: 
Theorem 4.3 (Homeomorphism Extension). Let $\varphi: C \rightarrow \mathbb{R}^{+}$and $\psi: D \rightarrow \mathbb{R}^{+}$ be Lelek functions with compact domain. Let $A \subset C$ and $B \subset D$ be closed sets such that $G_{0}^{\varphi \uparrow A}$ and $G_{0}^{\psi\lceil B}$ are nowhere dense in $G_{0}^{\varphi}$, respectively $G_{0}^{\psi}$. Then every homeomorphism from $\varphi \uparrow A$ to $\psi\lceil B$ can be extended to a homeomorphism from $\varphi$ to $\psi$ (with control).

Although it is a rather elegant characterization, Theorem 4.2 is not powerful enough because it is an 'external' or 'positional' characterization in two ways. First, in order to apply the theorem to an Erdös space candidate $E$ the space has to come equipped with a particular Lelek function $\varphi$ such that $E \approx G_{0}^{\varphi}$, that is, $E$ has to come positioned in the space $\mathbb{Q}^{\infty} \times \mathbb{R}^{+}$. In addition, any function $\varphi \in \mathrm{SL}$ assumes the value 0 (look for instance at $\eta$ ), which means that $E$ will always correspond to a proper subset of the graph of $\varphi$. Our next characterization will be 'internal'. In order to formulate the internal properties of a space that will guarantee the existence of Lelek functions such as $\varphi$ we need to introduce some new concepts.

As was mentioned in $\S 1$, Erdős [17 proved that every nonempty clopen subset of $\mathfrak{E}$ is unbounded. This means that every vector in $\mathfrak{E}$ has a neighbourhood that does not contain any nonempty clopen subsets of $\mathfrak{E}$. This property of $\mathfrak{E}$ turns out to be crucial, and we formalize it as follows.

Definition 4.4. Let $X$ be a space and let $\mathcal{A}$ be a collection of subsets of $X$. The space $X$ is called $\mathcal{A}$-cohesive if every point of the space has a neighbourhood that does not contain nonempty clopen subsets of any element of $\mathcal{A}$. If a space $X$ is $\{X\}$-cohesive, then we simply call $X$ cohesive.

A cohesive space is obviously at least one-dimensional at every point, but it is easily seen that the converse is not valid. However, the situation is simple for topological groups because a topological group is cohesive if and only if it is not zero-dimensional.

Definition 4.5. Let $T$ be a tree and let $\left(X_{s}\right)_{s \in T}$ be a system of subsets of a space $X$ such that $X_{t} \subset X_{s}$ whenever $s \prec t$. A subset $A$ of $X$ is called an anchor for $\left(X_{s}\right)_{s \in T}$ in $X$ if for every $\sigma \in[T]$ we have either $X_{\sigma \nmid k} \cap A=\emptyset$ for some $k \in \omega$ or the sequence $X_{\sigma\lceil 0}, X_{\sigma \uparrow 1}, \ldots$ converges to a point in $X$.

Thus the anchor $A$ has the property that for every sequence that is generated by an element of $[T]$, if it is attached to $A$, then it must converge and cannot be free to drift out of the space. Note that if $\left(X_{s}\right)_{s \in T}$ is a Sierpiński stratification, then the whole space is an anchor.

We now present our first internal characterization of $\mathfrak{E}$.

Definition 4.6. $\mathrm{E}$ is the class of all nonempty spaces $E$ such that there exists a topology $\mathcal{T}$ on $E$ that witnesses the almost zero-dimensionality of $E$ and there exist a nonempty tree $T$ over a countable set and subspaces $E_{s}$ of $E$ that are closed with respect to $\mathcal{T}$ for each $s \in T$ such that:

(1) $E_{\lambda}=E$ and $E_{s}=\bigcup\left\{E_{t}: t \in \operatorname{succ}(s)\right\}$ whenever $s \in T$,

(2) each $x \in E$ has a neighbourhood $U$ that is an anchor for $\left(E_{s}\right)_{s \in T}$ in $(E, \mathcal{T})$,

(3) for each $s \in T$ and $t \in \operatorname{succ}(s)$ we have that $E_{t}$ is nowhere dense in $E_{s}$, and

(4) $E$ is $\left\{E_{s}: s \in T\right\}$-cohesive.

Theorem 4.7. E is the class of all spaces that are homeomorphic to $\mathfrak{E}$. 
We sketch the essence of the proof. Let $E \in \mathrm{E}$ and let $\mathcal{T}$ and $\left(E_{s}\right)_{s \in T}$ be the associated witness topology and stratification. It suffices to show that there is a $\chi \in \mathrm{SL}$ with $E \approx G_{0}^{\chi}$. Let $Z$ denote the zero-dimensional space $(E, \mathcal{T})$. We begin by taking the stratification $\left(E_{s}\right)_{s \in T}$ through a 'refining' process such that $Z$ admits a zero-dimensional extension $X$ with the property that $\left(\bar{E}_{s}\right)_{s \in T}$ becomes a Sierpiński stratification of $X$, that is, the whole space becomes an anchor. Consider now the space $Y$ that corresponds to the set $X$ with the topology that is generated by the union of the topologies of $X$ and $E$. Then $X$ is a witness to the almost zero-dimensionality of $Y$ and $E$ is an open subspace of $Y$. With Theorem 3.1 we can find a USC function $\varphi$ the graph of which is homeomorphic to $Y$. By careful construction we can arrange that $\varphi: X \rightarrow \mathbb{I}$ and that $G_{0}^{\varphi} \approx E$. Condition (4) of Definition 4.6 allows us to construct a USC function $\psi: X \rightarrow \mathbb{I}$ such that $G_{0}^{\varphi}=G_{\psi}^{\varphi}$ and $\varphi \mid \bar{E}_{s}$ is a Lelek function with bias $\psi \mid \bar{E}_{s}$ for each $s \in T$. We then remove the bias by replacing the pair $(\varphi, \psi)$ by $(\chi, 0)$ such that $G_{\psi}^{\varphi} \approx G_{0}^{\chi}$ and $\chi\left\lceil\bar{E}_{s}\right.$ is a Lelek function for each $s \in T$. We now have that $\chi \in \mathrm{SL}$ and can apply Theorem 4.2

Remark 4.8. The anchor concept in Definition 4.6 is essential. This is because condition (4) excludes the possibility that the whole $E$ is an anchor for $\left(E_{s}\right)_{s \in T}$, that is, we cannot have a Sierpiński stratification.

Note that in our characterization theorems we need a particular witness topology on the space. Of course this topology is not uniquely determined. So one might ask why we do not use the 'witness topology' $\mathcal{T}$ that is generated by all clopen subsets of $E$. The reason is that this topology is not metrizable for Erdös space. Specifically, whenever $E$ is almost zero-dimensional and cohesive, then $\mathcal{T}$ has uncountable character at every point.

Our final and most powerful characterization of Erdős space is captured by the following definition.

Definition 4.9. $E^{\prime}$ is the class of all nonempty spaces $E$ such that there exists an $F_{\sigma \delta}$-topology $\mathcal{T}$ on $E$ that witnesses the almost zero-dimensionality of $E$ and there exist a nonempty tree $T$ over a countable set and subspaces $E_{s}$ of $E$ that are closed with respect to $\mathcal{T}$ for each $s \in T \backslash\{\lambda\}$ such that:

$\left(1^{\prime}\right) E_{\lambda}$ is dense in $E$ and $E_{s}=\bigcup\left\{E_{t}: t \in \operatorname{succ}(s)\right\}$ whenever $s \in T$,

$\left(2^{\prime}\right)$ each $x \in E$ has a neighbourhood $U$ that is an anchor for $\left(E_{s}\right)_{s \in T}$ in $(E, \mathcal{T})$,

$\left(3^{\prime}\right)$ for each $s \in T \backslash\{\lambda\}$ and $t \in \operatorname{succ}(s)$ we have that $E_{t}$ is nowhere dense in $E_{s}$,

$\left(4^{\prime}\right) E$ is $\left\{E_{s}: s \in T\right\}$-cohesive, and

$\left(5^{\prime}\right) E$ can be written as a countable union of nowhere dense subsets that are closed with respect to $\mathcal{T}$.

Theorem 4.10. $\mathrm{E}^{\prime}=\mathrm{E}=\{E: E \approx \mathfrak{E}\}$.

The inclusion $\mathrm{E} \subset \mathrm{E}^{\prime}$ is a triviality. Let us consider an element $E$ of $\mathrm{E}^{\prime}$ with associated topology $\mathcal{T}$ and stratification $\left(E_{t}\right)_{t \in T}$. Since $\mathcal{T}$ is an absolute $F_{\sigma \delta}$-topology we can find a Sierpiński stratification $\left(Z_{s}\right)_{s \in S}$ for $(E, \mathcal{T})$. The proof now consists in carefully 'grafting' the stratification $\left(E_{t}\right)_{t \in T}$ onto $\left(Z_{s}\right)_{s \in S}$ so that the combined stratification satisfies Definition 4.6,

Remark 4.11. At first glance there does not appear to be much difference between Definitions 4.6 and 4.9. This, however, is a false impression. To use Theorem 4.7 
we have to construct a stratification of the entire space, whereas condition $\left(1^{\prime}\right)$ of Definition 4.9 requires only a stratification of a dense subset of $E$. Let us examine the consequences if $E$ is for instance a topological group. Then we need only three things to satisfy Definition 4.9 an $F_{\sigma \delta}$ witness topology, the first category property $\left(5^{\prime}\right)$, and a suitable closed imbedding of Erdős space in $E$. Because if we have a copy $\mathcal{E}$ of $\mathfrak{E}$ in $E$ of the right type, which means in particular that it is also a closed imbedding on the level of the respective witness topologies, then we can obtain the dense stratified set $E_{\lambda}$ by simply multiplying $\mathcal{E}$ with a countable dense subset of the group $E$. This is the method that we will use to classify homeomorphism groups. In effect, Theorem 4.10 allows for a universality type argument similar to those used in zero-dimensional (cf. Corollary 2.2) and infinite-dimensional topology.

\section{Applications}

The following result follows easily from Theorem 4.7 .

Proposition 5.1. If $A \subset \mathfrak{E}$ is either nonempty and open or the complement of a $\sigma$-compactum, then $A$ is homeomorphic to $\mathfrak{E}$.

The following lemma can be found in Dijkstra [10] and is a straightforward generalization of Erdös [17. Recall that if $A_{0}, A_{1}, \ldots$ is a sequence of subsets of a space $X$, then $\lim \sup _{n \rightarrow \infty} A_{n}=\bigcap_{n=0}^{\infty} \overline{\bigcup_{k=n}^{\infty} A_{k}}$. Consider the space $\ell^{p}$.

Lemma 5.2. Let $E_{0}, E_{1}, E_{2}, \ldots$ be a sequence of subsets of $\mathbb{R}$ such that 0 is a cluster point of $\limsup _{n \rightarrow \infty} E_{n}$. If we define

$$
\mathcal{E}=\left\{z \in \ell^{p}: z_{n} \in E_{n} \text { for every } n\right\},
$$

then every nonempty clopen subset of $\mathcal{E}$ is unbounded (and hence $\mathcal{E}$ is cohesive).

The following results show that there is great flexibility in the construction of $\mathfrak{E}$.

Proposition 5.3. Let $\mathcal{E}$ be a nonempty space as in Lemma 5.2 such that every $E_{n}$ is an $F_{\sigma \delta}$-space that is zero-dimensional. If infinitely many of the $E_{n}$ 's are of the first category in themselves, then $\mathcal{E} \in \mathrm{E}$. Thus $\mathcal{E}$ is homeomorphic to $\mathbb{E}$.

Corollary 5.4. $\mathfrak{E}_{\mathrm{c}} \times \mathbb{Q}^{\infty}$ is homeomorphic to $\mathfrak{E}$.

A space $X$ is called an Erdös space factor if there exists a space $Y$ with $X \times Y \approx$ $\mathfrak{E}$. The following characterization follows easily from Theorem 4.7.

Theorem 5.5. For a nonempty space $E$ the following statements are equivalent:

(1) $E \times \mathfrak{E}$ is homeomorphic to $\mathfrak{E}$,

(2) $E$ is an Erdös space factor,

(3) E admits a closed imbedding into $\mathfrak{E}$,

(4) $E$ is homeomorphic to a $G_{\delta}$-subset of $\mathfrak{E}$, and

(5) $E$ is almost zero-dimensional as witnessed by an $F_{\sigma \delta}$-topology.

Corollary 5.6. $\mathfrak{E}^{\infty}$ is homeomorphic to $\mathfrak{E}$.

Our main applications are the ones on homeomorphism groups mentioned in $\$ 1$ The first step in satisfying Definition 4.9 is to find an $F_{\sigma \delta}$ witness topology. If $D$ is a countable dense subset of a compact space $X$, then the topology of pointwise convergence on $D$ turns out to be precisely the right witness to the almost zerodimensionality of $\mathcal{H}(X, D)$. In view of Theorem 5.5 we now have: 
Theorem 5.7. If $D$ is a countable dense subset of a locally compact space $X$, then $\mathcal{H}(X, D)$ is an Erdös space factor.

We use Theorem 4.10 to prove our main application, as follows.

Theorem 5.8. Let $D$ be a countable dense subset of a locally compact space $X$. If $X$ contains an open set that is a topological $n$-manifold with $n \geq 2$, a Hilbert cube manifold, or a manifold modelled on a universal Menger continuum, then $\mathcal{H}(X, D)$ is homeomorphic to Erdös space.

To use the method outlined in Remark 4.11 we need suitable imbeddings of Erdős space in $\mathcal{H}(X, D)$. Fortunately, when we arrived at this point, it turned out that we already constructed the right imbeddings in Dijkstra and van Mill [12] and Dijkstra [10] for the purpose of showing that the homeomorphism groups in question are one-dimensional.

\section{REFERENCES}

[1] S. Ageev, A characterization of $k$-dimensional Nöbeling space for every $k>1,2002$ preprint.

[2] R. D. Anderson, Spaces of homeomorphisms of finite graphs, unpublished manuscript.

[3] R. Arens, Topologies for homeomorphism groups, Amer. J. Math. 68 (1946), 593-610. MR 8:479i

[4] C. Bessaga and A. Pełczyński, Selected topics in infinite-dimensional topology, Mathematical Monographs, Vol. 58, PWN-Polish Scientific Publishers, Warsaw, 1975. MR 57:17657

[5] M. Bestvina, Characterizing $k$-dimensional universal Menger compacta, Mem. Amer. Math. Soc. 71 (1988), no. 380. MR 89g:54083

[6] L. E. J. Brouwer, On the structure of perfect sets of points, Proc. Akad. Amsterdam 12 (1910), 785-794.

[7] L. E. J. Brouwer, Some remarks on the coherence type $\eta$, Proc. Akad. Amsterdam 15 (1913), $1256-1263$.

[8] W. D. Bula and L. G. Oversteegen, A characterization of smooth Cantor bouquets, Proc. Amer. Math. Soc. 108 (1990), 529-534. MR 90d:54066

[9] W. J. Charatonik, The Lelek fan is unique, Houston J. Math. 15 (1989), 27-34. MR 90f: 54050

[10] J. J. Dijkstra, On homeomorphism groups of Menger continua, 2003 preprint.

[11] J. J. Dijkstra, On homeomorphism groups and the compact-open topology, 2003 preprint.

[12] J. J. Dijkstra and J. van Mill, On the group of homeomorphisms of the real line that map the pseudoboundary onto itself, 2002 preprint.

[13] J. J. Dijkstra and J. van Mill, Erdös space and homeomorphism groups of manifolds, in preparation.

[14] J. J. Dijkstra, J. van Mill, and J. Steprāns, Complete Erdös space is unstable, Math. Proc. Cambridge Philos. Soc., to appear.

[15] T. Dobrowolski and H. Toruńczyk, Separable complete ANRs admitting a group structure are Hilbert manifolds, Topology Appl. 12 (1981), 229-235. MR 83a:58007

[16] A. J. M. van Engelen, Homogeneous zero-dimensional absolute Borel sets, CWI Tract, Vol. 27, Centre for Mathematics and Computer Science, Amsterdam, 1986. MR 87j:54058

[17] P. Erdős, The dimension of the rational points in Hilbert space, Ann. of Math. 41 (1940), 734-736. MR 2:178a

[18] S. Ferry, The homeomorphism group of a compact Hilbert cube manifold is an ANR, Ann. of Math. 106 (1977), 101-119. MR 57:1521

[19] K. Kawamura, L. G. Oversteegen, and E. D. Tymchatyn, On homogeneous totally disconnected 1-dimensional spaces, Fund. Math. 150 (1996), 97-112. MR 97d:54060

[20] J. E. Keesling, Using flows to construct Hilbert space factors of function spaces, Trans. Amer. Math. Soc. 161 (1971), 1-24. MR 44:981

[21] A. Lelek, On plane dendroids and their end points in the classical sense, Fund. Math. 49 (1961), 301-319. MR 24:A363

[22] M. Levin and R. Pol, A metric condition which implies dimension $\leq 1$, Proc. Amer. Math. Soc. 125 (1997), 269-273. MR 97e:54033 
[23] R. Luke and W. K. Mason, The space of homeomorphisms of a compact two-manifold is an Absolute Neighborhood Retract, Trans. Amer. Math. Soc. 164 (1972), 275-285. MR 46:849

[24] L. G. Oversteegen and E. D. Tymchatyn, On the dimension of certain totally disconnected spaces, Proc. Amer. Math. Soc. 122 (1994), 885-891. MR 95b:54040

[25] R. Pol, There is no universal totally disconnected space, Fund. Math. 70 (1973), 265-267. MR 48:1139

[26] W. Sierpiński, Sur une définition topologique des ensembles $F_{\sigma \delta}$, Fund. Math. 6 (1924), $24-29$.

[27] J. R. Steel, Analytic sets and Borel isomorphisms, Fund. Math. 108 (1980), 83-88. MR 82b:03091

[28] H. Toruńczyk, Homeomorphism groups of compact Hilbert cube manifolds which are manifolds, Bull. Acad. Polon. Sci. Sér. Sci. Math. Astronom. Phys. 25 (1977), 401-408. MR 58:24302

[29] H. Toruńczyk, On CE-images of the Hilbert cube and characterizations of Q-manifolds, Fund. Math. 106 (1980), 31-40. MR 83g:57006

[30] H. Toruńczyk, Characterizing Hilbert space topology, Fund. Math. 111 (1981), 247-262. MR 82i: 57016

Faculteit der Exacte Wetenschappen / Afdeling Wiskunde, Vrije Universiteit, De Boelelaan 1081, 1081 HV Amsterdam, The Netherlands

E-mail address: dijkstra@cs.vu.nl

Faculteit der Exacte Wetenschappen / Afdeling Wiskunde, Vrije Universiteit, De Boelelaan 1081, $1081 \mathrm{HV}$ Amsterdam, The Netherlands

E-mail address: vanmill@cs.vu.nl 